\title{
Is there any geometrical information in the nervous system?
}

\section{Sajad Jafari *, Seyed M. R. Hashemi Golpayegani and Shahriar Gharibzadeh}

Biomedical Engineering Faculty, Amirkabir University of Technology, Tehran, Iran

${ }^{*}$ Correspondence: sajadjafari@aut.ac.ir

Edited by:

Tobias A. Mattei, Ohio State University, USA

Keywords: trains of impulses, chaotic systems, sensitivity to initial conditions, geometry, phase space

There has been an increasing interest in analyzing neurophysiology from complex and chaotic systems viewpoint in recent years. For example, although the famous Hodgkin and Huxley model (Hodgkin and Huxley, 1952) has been the basis of almost all of the proposed models for neural firing, the Rose-Hindmarsh model (Hindmarsh and Rose, 1984) is known to be a more refined model because as it has the ability of showing different firing patterns, especially chaotic bursts of action potential, which causes a proper matching between this model behavior and many real experimental data.
It is believed that information is transferred in the brain by trains of impulses, or action potentials, often organized in sequences of bursts; therefore, it is useful to determine the temporal patterns of such trains (Korn and Faure, 2003). Since chaotic systems are sensitive to initial conditions (Hilborn, 2000), lots of signals with minimum similarity in time domain could have a same source; such behavior might be better understood by analyzing those signals in the phase space and from geometrical viewpoint (Jafari et al., 2013d), as although chaotic signals have pseudorandom behavior in time, they are
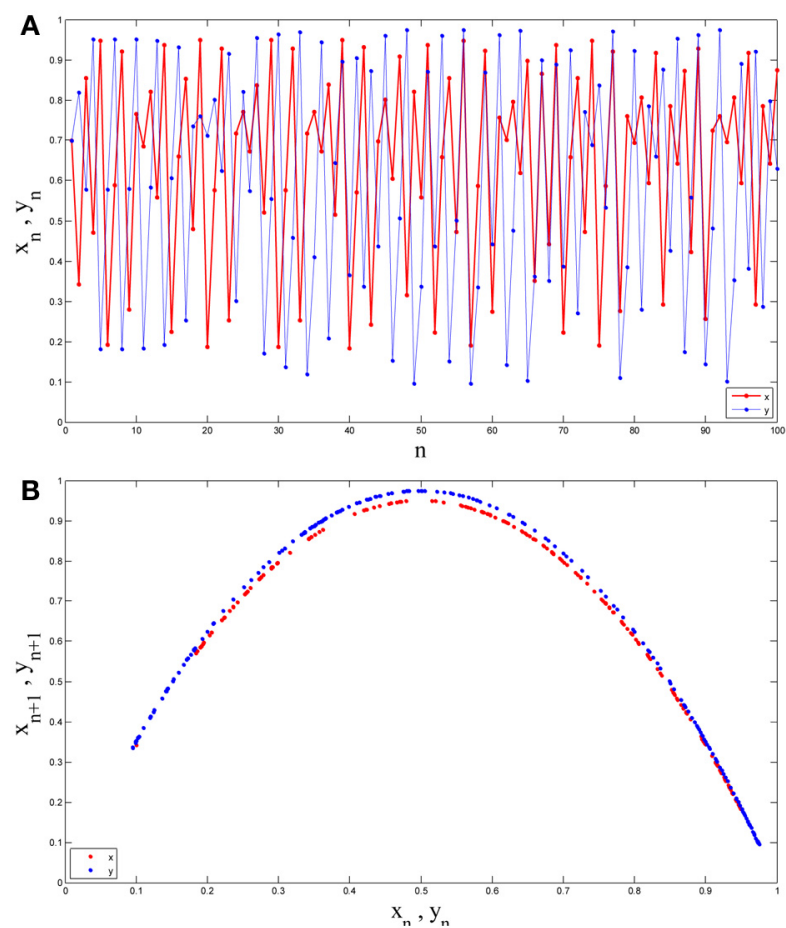

FIGURE 1 | (A) Two time series obtained from two different Logistic maps. (B) Those two time series embedded in the state space. As can be seen while recognizing the difference between them is not such easy in the time domain (both are random-like), they have two ordered and easily distinguishable pattern in the state space.

ordered in phase space (i.e., if one plots the signals as a trajectory in a coordinate of system variables, he will encounter an ordered and specific topology which is called strange attractor) (Hilborn, 2000).

In fact in many applications of chaotic signals and systems, using temporal properties without being careful about this sensitivity to initial conditions, could lead to important misinterpretations (Jafari et al., 2012, 2013a,c,d). Hence, it seems that more than temporal patterns, it is of paramount importance to investigate topological patterns in such impulse trains. In order to accomplish such tasks several we have recently proposed some interesting tools for geometrical analysis (Jafari et al., in press; Shekofteh et al., in press).

In order to show the benefit of using geometry and topology in the phase space (state space), a simple example is provided in the sequence. Consider the famous Logistic map which is a very simple and well investigated chaotic map:

$$
x_{k+1}=A x_{k}\left(1-x_{k}\right)
$$

Suppose that we have two different maps with different values of parameter A:

$$
\begin{aligned}
& x_{k+1}=3.8 x_{k}\left(1-x_{k}\right) \\
& x_{k+1}=3.9 x_{k}\left(1-x_{k}\right)
\end{aligned}
$$

If we obtain one time series from each of them, as can be seen in Figure 1A, they are both random-like and recognizing the difference between them seems difficult in the time domain. However, they have two ordered and easily distinguishable patterns in the state space (Figure 1B).

Since looking at neurophysiology from dynamical and geometrical points of view has already been successfully investigated 
in some previous works (Sauer, 1994; Christini and Collins, 1995; Gottschalk et al., 1995; Milton and Black, 1995; Sarbadhikari and Chakrabarty, 2001; Korn and Faure, 2003; Hadaeghi et al., 2013; Jafari et al., 2013a), we believe that future investigations, especially using real clinical data, will be able evaluate our hypothesis and prove the benefit of such geometrical analysis of non-linear data. Ultimately, a better understanding of neuronal information transportation from the nonlinear dynamics standpoint is expected to provide a better understanding of the basic pathophysiology of neurological disorders, possibly fostering new future therapeutic approaches.

\section{REFERENCES}

Christini, D. J., and Collins, J. J. (1995). Controlling nonchaotic neuronal noise using chaos control techniques. Phys. Rev. Lett. 75, 2782-2785. doi: 10.1103/PhysRevLett.75.2782

Gottschalk, A., Bauer, M. S., and Whybrow, P. C. (1995). Evidence of chaotic mood variation in bipolar disorder. Arch. Gen. Psychiatry 52, 947-959. doi: 10.1001/archpsyc.1995. 03950230061009

Hadaeghi, F., Hashemi Golpayegani, M., and Moradi, K. (2013). Does "Crisis-Induced Intermittency" explain bipolar disorder dynamics. Front. Comput. Neurosci. 7:116. doi: 10.3389/fncom.2013.00116

Hilborn, R. C. (2000). Chaos and Nonlinear Dynamics, 2nd Edn. New York, NY: Oxford University Press Inc. doi: 10.1093/acprof:oso/9780198507239.001. 0001
Hindmarsh, J. L., and Rose, R. M. (1984). A model of neuronal bursting using three coupled first order differential equations. Proc. R. Soc. Lond. B Biol. Sci. 221, 87-102. doi: 10.1098/rspb.1984.0024

Hodgkin, A. L., and Huxley, A. F. (1952). A quantitative description of membrane current and its application to conduction and excitation in nerve. J. Physiol. 117, 500-544.

Jafari, S., Baghdadi, G., Hashemi Golpayegani, S. M. R., Towhidkhah, F., and Gharibzadeh, S. (2013a). Is attention deficit hyperactivity disorder a kind of intermittent chaos. J. Neuropsychiatry Clin. Neurosci. 25:E02. doi: 10.1176/appi.neuropsych.12040079

Jafari, S., Hashemi Golpayegani, S. M. R., and Daliri, A. (2013b). Comment on 'Parameters identification of chaotic systems by quantum-behaved particle swarm optimization' [Int. J. Comput. Math. 86 (12) (2009), pp. 2225-2235]. Int. J. Comput. Math. 90, 903-905. doi: 10.1080/00207160.2012.743651

Jafari, S., Hashemi Golpayegani, S. M. R., and Darabad, M. R. (2013c). Comment on "Parameter identification and synchronization of fractionalorder chaotic systems" (Commun Nonlinear Sci Numer Simulat 2012;17:305-16). Commun. Nonlinear Sci. Numer. Simulat. 18, 811-814. doi: 10.1016/j.cnsns.2012.07.020

Jafari, S., Hashemi Golpayegani, S. M. R., Jafari, A. H., and Gharibzadeh, S. (2013d). A novel viewpoint on the parameter estimation in a chaotic neuron model. J. Neuropsychiatry Clin. Neurosci. 25:E19. doi: 10.1176/appi.neuropsych.12010012

Jafari, S., Hashemi Golpayegani, S. M. R., Sprott, J. C., Jafari, A. H., and Abdolmohammadi, H. R. (in press). A new cost function for parameter estimation of chaotic maps. Int. J. Bifurcation Chaos.

Jafari, S., Hashemi Golpayegani, S. M. R., Jafari, A. H., and Gharibzadeh, S. (2012). Some remarks on chaotic systems. Int. J. Gen. Syst. 41, 329-330. doi: 10.1080/03081079.2012.664855
Korn, H., and Faure, P. (2003). Is there chaos in the brain. II. Experimental evidence and related models. C. R. Biol. 326, 787-840. doi: 10.1016/j.crvi.2003.09.011

Milton, J., and Black, D. (1995). Dynamic diseases in neurology and psychiatry. Chaos 5, 8-13. doi: $10.1063 / 1.166103$

Sarbadhikari, S. N., and Chakrabarty, K. (2001). Chaos in the brain: a short review alluding to epilepsy, depression, exercise and lateralization. Med. Eng. Phys. 23, 445-455. doi: 10.1016/S13504533(01)00075-3

Sauer, T. (1994). Reconstruction of dynamical systems from interspike intervals. Phys. Rev. Lett. 72, 3811-3814. doi: 10.1103/PhysRevLett.72.3811

Shekofteh, Y., Jafari, S., Sprott, J. C., Hashemi Golpayegani, S. M. R., and Almasganj, F. (in press). A gaussian mixture model based cost function for parameter estimation of chaotic biological systems. Commun. Nonlinear Sci. Numer. Simulat.

Received: 03 August 2013; accepted: 15 August 2013; published online: 30 August 2013.

Citation: Jafari S, Hashemi Golpayegani SMR and Gharibzadeh S (2013) Is there any geometrical information in the nervous system? Front. Comput. Neurosci. 7:121. doi: 10.3389/fncom.2013.00121

This article was submitted to the journal Frontiers in Computational Neuroscience.

Copyright (c) 2013 Jafari, Hashemi Golpayegani and Gharibzadeh. This is an open-access article distributed under the terms of the Creative Commons Attribution License (CC BY). The use, distribution or reproduction in other forums is permitted, provided the original author(s) or licensor are credited and that the original publication in this journal is cited, in accordance with accepted academic practice. No use, distribution or reproduction is permitted which does not comply with these terms. 